EPJ Web of Conferences 92,02100 (2015)

DOI: $10.1051 /$ epjconf/ 20159202100

(C) Owned by the authors, published by EDP Sciences, 2015

\title{
Modelling fluid flow in a reciprocating compressor
}

\author{
Jan Tuhovcak ${ }^{1, a}$ Jiří Hejčík ${ }^{1}$ and Miroslav Jícha ${ }^{1}$ \\ ${ }^{I}$ Brno University of Technology, Faculty of Mechanical Engineering, Energy Institute, Technicka 2896/2 Brno 616 69, \\ Czech Republic
}

\begin{abstract}
Efficiency of reciprocating compressor is strongly dependent on the valves characteristics, which affects the flow through the suction and discharge line. Understanding the phenomenon inside the compressor is necessary step in development process. Commercial CFD tools offer wide capabilities to simulate the flow inside the reciprocating compressor, however they are too complicated in terms of computational time and mesh creation. Several parameters describing compressor could be therefore examined without the CFD analysis, such is valve characteristic, flow through the cycle and heat transfer. The aim of this paper is to show a numerical tool for reciprocating compressor based on the energy balance through the cycle, which provides valve characteristics, flow through the cycle and heat losses from the cylinder. Spring-damping-mass model was used for the valve description. Boundary conditions were extracted from the performance test of 4cylinder semihermetic compressor and numerical tool validation was performed with indicated $p$ - $V$ diagram comparison.
\end{abstract}

\section{Introduction}

Reciprocating compressors are commonly used in many technical fields, from household appliances to large-scale compressors used in refineries and gas engineering. Reducing compressor energy consumption by improving the efficiency is a relevant step in decreasing the global energy consumption. To achieve this goal it is necessary to understand processes inside the compressor: heat flux and fluid flow. Analysis according to [1] shows that contribution to overall electric energy consumption in US reaches up to $8 \%$. Statistics from industry sector are not known, however expectation are even higher. Overall efficiency of compressor consist of three main subefficiencies: electrical, mechanical and thermodynamic efficiency. Thermodynamic efficiency is the lowest $(80-$ $83 \%)$, therefore there is significant effort to improve parts of compressor, which have the biggest influence on the efficiency: pressure losses in suction and discharge line, superheating of gas and cylinder leakage. Three dimensional (3D) numerical tools (CFD) offer the most detailed method for thermodynamic analysis of compressor, however they still have high demands on computational power and time, even despite progress in computer science in last years. As this approach is not suitable in development process, several simplified models were developed to analyze reciprocating compressor. Basically, there are three approaches used in analysis of compressors. Quasi-static (0D) simulation tool uses the energy balance over a control volume [2]. More complicated numerical tool was introduced by [3], solving Euler equations over a cross section perpendicular to piston in 1D model or over the height of the cylinder in $2 \mathrm{D}$ model. The last option of zooming into a compressor is abovementioned numerical tool based on solving Navier-Stokes equation over a control volume. In this paper 0D model is presented for in-cylinder analysis of the compressor.

\section{The model}

Analysis of reciprocating compressor with 0D model, presented in this paper, can be used to evaluate following phenomena:

- Pressure history in a cylinder

- Valve dynamics and motion

- Pressure losses of a valve

The energy analysis of unsteady flow serve as a base for $0 \mathrm{D}$ model. Flow inside the cylinder is neglected and pressure is considered as uniform in the cylinder as well as temperature, calculated using of the $1^{\text {st }}$ Law of Thermodynamics,

$$
d W+d Q+\sum_{i} d m_{i} \cdot h_{i}=d U .
$$

$Q$ stands for heat transfer from/to the cylinder. Inflow or outflow through the valves is represented by $d m_{i} h_{i}$ and the change of inner energy is $d U$. The mass balance in the control volume is described by eq. (2), where $m_{c y l}$ is mass inside the cylinder and $m_{i}$ is mass transported over valves.

$$
\frac{d m_{c y l}}{d t}+\sum_{i} m_{i}=0 \text {. }
$$

\footnotetext{
${ }^{\mathrm{a}}$ Corresponding author: tuhovcak@fme.vutbr.cz
} 


\subsection{Crank mechanism}

The work of the piston, $d W$, is done by the change of a volume, which is determined by a piston motion. Using the properties of the crank mechanism: length of the crank lever $r$, the length of the piston rod $l$ and the smallest distance between a piston and a cylinder $S_{0}$ (derived from the clearance volume), movement of the piston could be solved for present angle of crankshaft in time $\alpha$, eq. (3).

$$
S=r+l+S_{0}-r \cdot \cos \alpha-l \sqrt{l-\left(\frac{r}{l}\right)^{2} \sin ^{2} \alpha} .
$$

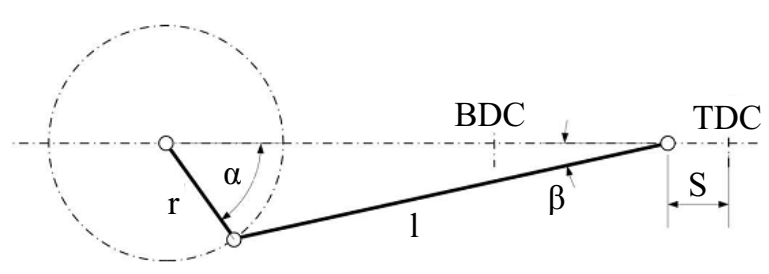

Figure 1. Crank mechanism parameters.

\subsection{Mathematical model of valves}

Valves inside the compressor are operated by the forces acting on it. A mathematical model was introduced by [4] as a mass-spring system, examined more deeply in the work of [5]. Movement of the valve can be described by single degree of freedom equation

$$
\begin{gathered}
m \cdot \ddot{x}+d \cdot \dot{x}+k \cdot x=\sum_{i} F_{i}, \\
\dot{x}_{(n-1)}=-e \cdot \dot{x}_{(n)} .
\end{gathered}
$$

Mass of the valve $m$ and stiffness of the spring $k$ are basic properties of the spring system, however the damping constant $d$ must be determined experimentally, as it is formed not just by the system, but also with the surrounding environment. Usually it is left from eq. (4) as its value is negligible [3]. When the valve hits the limiter, it loses part of the kinetic energy, expressed by restitution coefficient $e$ in eq. (5). Significant contribution to the force acting on valve have pressure difference over the valve, stiffness of the spring and adhesive forces due to oil between valve surface and seating. Adhesive force $F_{\text {adh }}$ causes delay in valve opening and its approximated value could be obtained by using eq.(6) [3].

$$
F_{a d h}=f \cdot \frac{\dot{x}}{x^{3}} \text {. }
$$

Parameter $f$ stands for geometry properties of the valve and seating. Equation (4) is solved by using $4^{\text {th }}$ order Runge-Kutta method.

\subsection{Flow through the valve}

Fliegner's equation is used to calculate flow through the nozzle. The flow is considered as stationary, one dimensional and isentropic.

$$
\dot{m}=\phi_{\text {eff }} \cdot \rho_{1} \cdot\left(\frac{p_{2}}{p_{1}}\right)^{\frac{1}{\kappa}} \sqrt{\frac{2 \kappa}{\kappa-1} \frac{p_{1}}{\rho_{1}}\left(1-\left(\frac{p_{2}}{p_{1}}\right)^{\frac{\kappa-1}{\kappa}}\right)} .
$$

Effective flow area $\varphi_{\text {eff }}$ includes besides actual flow area, determined from valve position as a geometry function, also local pressure loss coefficient $\zeta$. All other parameters have their usual meaning.

\subsection{Heat Transfer}

Heat transfer in the cylinder influences the overall efficiency of compressor significantly. Heating up the working fluid at the beginning of compression process by $1 \mathrm{~K}$ will lead to reduction of COP by $0.32 \%$ [6]. Several approaches were used in relevant literature to evaluate heat transfer coefficient correctly, however they seem to under-predict or over-predict the value, especially during the suction or discharge process. Most significant of them were analyzed by Pereira [7], although none of them agreed with his CFD simulation of simplified axisymmetric domain. All approaches were based on correlation of Nusselt number with different constants $a$, $b$ and $c$,

$$
N u=a \operatorname{Re}^{b} \operatorname{Pr}^{c} .
$$

Determining the velocity inside the compressor plays important role in the compressor heat transfer model. The velocity is given by a piston speed during the compression. Discharge and suction process are more complicated as the velocity of the fluid behind the valve is much higher than in piston speed and is strongly

\begin{tabular}{|c|c|c|}
\hline Process & Reynolds number & $\begin{array}{c}\text { Constants } \\
\text { a } \\
\text { b } \\
\text { c }\end{array}$ \\
\hline Compression & $\operatorname{Re}=\frac{\rho_{(t)} D V_{P}}{\mu_{(t)}}$ & $\begin{array}{c}0.08 \\
0.8 \\
0.6\end{array}$ \\
\hline Discharge & $\operatorname{Re}=\frac{\rho_{(t)} D V_{P}+V_{P}^{0.8} V_{C(t)}^{0.2}}{\mu_{(t)}}$ & $\begin{array}{c}0.08 \\
0.8 \\
0.6\end{array}$ \\
\hline Expansion & $\operatorname{Re}=\frac{\rho_{(t)} D V_{P}}{\mu_{(t)}}$ & $\begin{array}{c}0.12 \\
0.8 \\
0.6\end{array}$ \\
\hline Suction & $\operatorname{Re}=\frac{\rho_{(t)} D V_{P}+V_{P}^{-0.4} V_{C(t)}^{1.4}}{\mu_{(t)}}$ & $\begin{array}{c}0.08 \\
0.9 \\
0.6\end{array}$ \\
\hline
\end{tabular}
affected by manifold geometry. Disconzi [8] suggested to divide the compressor cycle in four processes: intake, compression, expansion and exhaust.

Table 1. Reynolds number and constants for processes inside the cylinder

For each process he proposed new correlation for heat transfer based on numerical simulation (Table 1). New correlation was also proposed to calculate velocity used in Reynolds number. During the suction and discharge 
process it is derived from the mass flow rate through the valve:

$$
V_{c}=\frac{\left|\dot{m}_{(t)}\right|}{\rho_{(t)} \cdot A_{c}} .
$$

Where $\rho_{(t)}$ is the density of gas in the cylinder and $A_{c}$ is the cross-sectional area of the cylinder. $V_{p}$ stands for the piston velocity. Table 1 shows new correlations from the work of Disconzi. He noted that the highest amount of heat is transferred during the suction due to the high temperature difference between sucking gas and cylinder walls. Also the heat transfer area is significantly larger compared to discharge, which increases the importance of correct heat transfer prediction in cylinder.

\subsection{Pressure waves in the cylinder}

Distribution of pressure inside the cylinder is not completely uniform and pressure waves occur when the valves open and thus interact with the outflow valve. Pressure waves are present in particular compressors type as well as during re-expansion, even during the compression process [3]. In reciprocating compressor they cause negative effect on the crank mechanism, exciting an oscillating moment onto the piston. Second effect is associated with a valve movement. Analysis of Aigner [3] concluded that valve dynamics is not correctly described by conventional methods, when the pressure waves are significant inside the cylinder. Impact velocity of valves is overestimated, which is important fact in design process of a compressor. Moreover the losses through the valve are increased, reducing the overall efficiency. However, when the piston velocity is small compared to the velocity of sound in particular environment and the motion is sufficiently smooth the state of the gas in cylinder can be described by quasistatic change of state $-0 \mathrm{D}$ model [9]. Nevertheless, the waves initiated by valves could be still present, especially with compressing heavy gases in fast running compressors. Therefore Steinrueck [9] introduced dimensionless classification number $\varepsilon$ as a ratio of characteristic time scales.

$$
\begin{gathered}
\varepsilon=\frac{\Delta t_{w}}{\Delta t_{D}}, \\
\Delta t_{w}=\frac{d}{c_{i}}, \\
\Delta t_{D}=\sqrt{\frac{S_{0}}{d^{2} S / d t^{2}} .}
\end{gathered}
$$

Time scale $t_{w}$ expresses the time that pressure wave needs to pass through the diameter of the cylinder. Characteristic dimension - diameter is chosen due to valves located around the cylinder in this case. Using the compressor with valves located on the top of the cylinder changes diameter to the bore of the cylinder. Acceleration of the piston in the top dead center (TDC), where it has maximum, is represented by time scale $t_{D}$. When the classification number $\varepsilon<<1$, quasi-static model is valid.

\subsection{Compressor}

The simulations were carried out for a real compressor, from which we took geometry parameters and estimated valve properties (Table 2). The working fluid for compressors depends on its purpose. Ideal air was in presented simulation tool to simplify the model in its development phase. Boundary conditions (BC) were obtained from real compressor measurements and also geometry parameters are similar to the compressor.

Table 2. Compressor specification

\begin{tabular}{|c|c|}
\hline Parameter & Dimension \\
\hline Diameter of cylinder bore & $80 \mathrm{~mm}$ \\
\hline Crank height & $28.75 \mathrm{~mm}$ \\
\hline Rod length & $115 \mathrm{~mm}$ \\
\hline Stroke & $57 \mathrm{~mm}$ \\
\hline Crankshaft speed & $1450 \mathrm{rpm}$ \\
\hline Clearance volume & $3.78 \times 10^{3} \mathrm{~mm}^{3}$ \\
\hline Valve weight & $0.05 \mathrm{~kg}$ \\
\hline Spring stiffness & $5000 \mathrm{~N}$ \\
\hline Damping constant & $0.1 \mathrm{Ns}$ \\
\hline
\end{tabular}

When the pressure exerts a force on the valve, it moves to certain position dependent on pressure difference magnitude and the stiffness of the spring. Flow area is calculated from the valve position and geometrical function describing the shape of valve seat.

Boundary conditions (Table 3 ) were used as for real compressor, extracted from the experiments.

Table 3. Boundary conditions used in the simulation tool

\begin{tabular}{|c|c|}
\hline Boundary condition & Value \\
\hline Suction pressure & $4.36 \mathrm{bar}$ \\
\hline Discharge pressure & $20.5 \mathrm{bar}$ \\
\hline Inlet temperature & $20^{\circ} \mathrm{C}$ \\
\hline
\end{tabular}

\section{Methodology}

The compressor simulation tool is solving the energy eq. (1) transferred from differential form to difference equation. Pressure inside the cylinder is linearly approximated. Choosing the correct time step is essential for simulation tool accuracy.

\section{Results}

The simulation tool offers an overview on compressor working process and its efficiency. Different working condition and compressor geometries can be evaluated with the tool, however exact $\mathrm{BC}$ and valves description is a necessary input. We used only estimated values as BC, e.g. for valves, especially pressure loss coefficients for presented results. Therefore the results are not comparable with an actual compressor, however the trends are correct. 
Fig. 2 shows the pressure behavior in the cylinder over two crankshaft cycles. To overcome the stiffness of the spring and the adhesive force of the oil the pressure inside the cylinder raises up to 29 bars. Decrease in the cylinder pressure during the suction process is not as significant as for the discharge process due to the different valve characteristic used in the model.

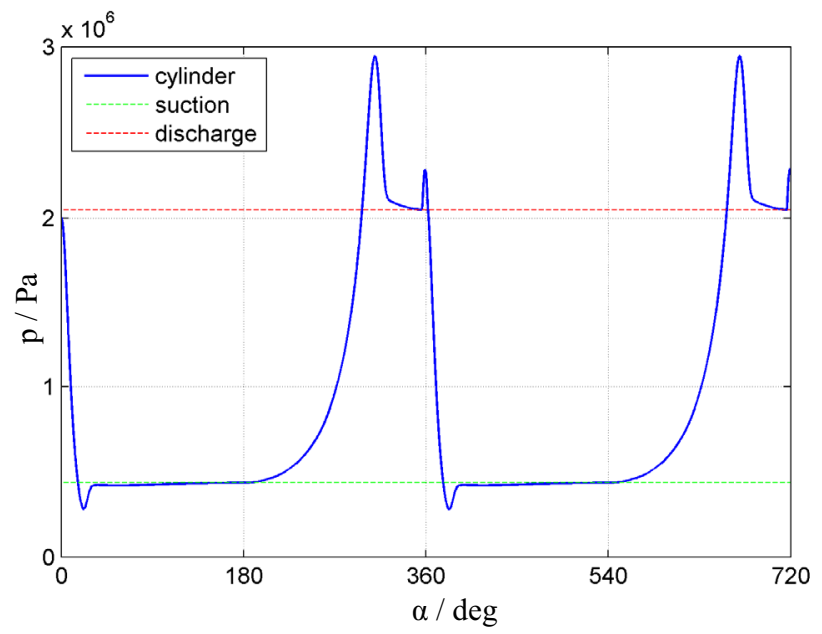

Figure 2. Pressure history inside the cylinder.

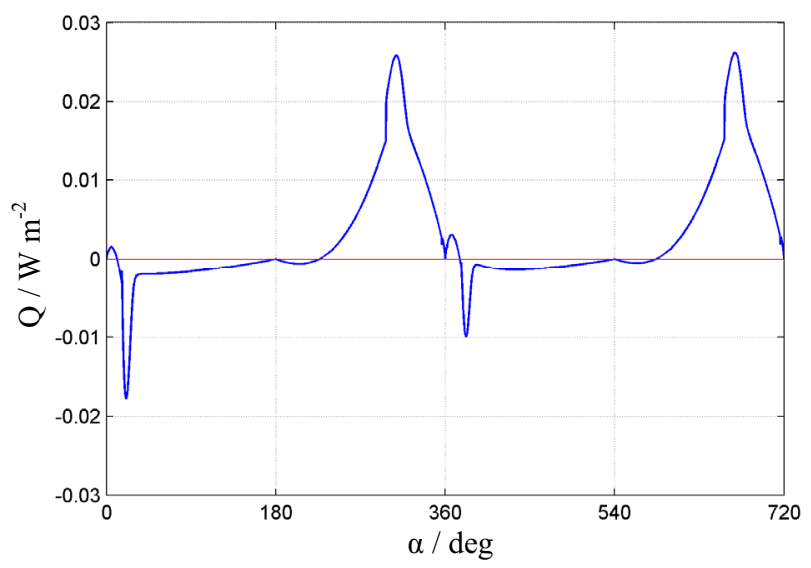

Figure 3. Heat flux from/to the cylinder.

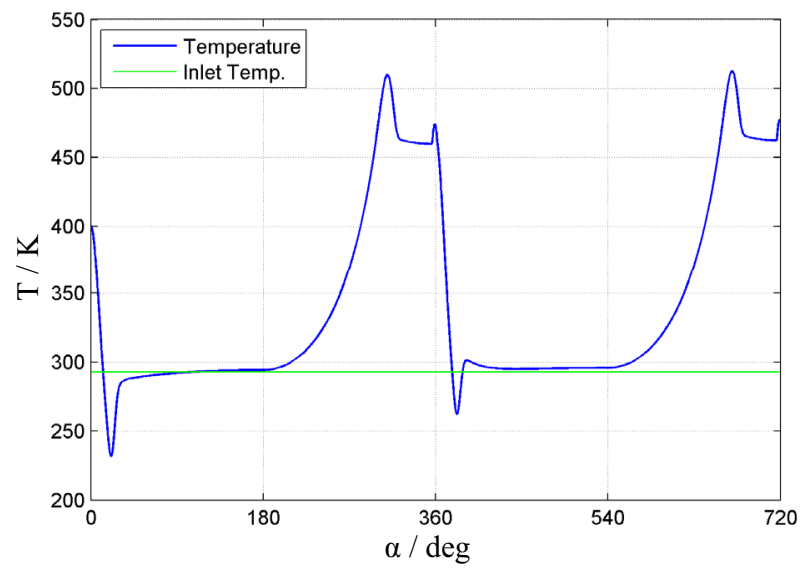

Figure 4. Temperature history through the cycle.

Motion of valves could be evaluated also from the Fig. 2. Valves are opened when the pressure before the valve is higher than behind it. Valves are moving back to the closed position rather fast and the oscillation of valves is not present, which is not usual in real situations and the reason can be found in the inaccurate valve description. The pressure loss is changing with the valve movement, however the model uses only one value for pressure loss and the actual flow area is changing in time.

The heat transfer inside the cylinder was analyzed using Diconzi's correlation. He proposed a correlation for each process inside the cylinder. Therefore the heat transfer during the cycle has no smooth history. When the process inside the cylinder changes, also the heat transfer equation changes, causing sudden changes in the graph (Fig. 3.) Overall behavior is similar to a real compressor, however the solution shows inaccuracies. During the suction the heat is transferred from the cylinder wall to the gas, while during the latter phase of compression and discharge the heat is transferred in opposite direction. The temperature difference wall - gas is smaller during the suction than during the discharge, however the heat transfer surface is much bigger. The rapid change of transferred heat during the suction process or discharge is caused by mass flow coming to / from cylinder.

Temperature history is shown in the Fig. 4. As the gas is compressed, an increase in the temperature is present. After the re-expansion the temperature decreases even under the suction temperature, what is theoretically possible, but in reality not a usual state.

\section{Conclusion}

The 0D simulation tool for reciprocating compressor was developed in the present study. The model is capable of analyzing basic compressor parameters, evaluating the valve behavior, pressure history and heat transfer in the cylinder. Because of missing experimental data the model was not validated for real compressor.

\section{Acknowledgement}

This work was supported by the project RP9042100306/1120 of Brno University of Technology Faculty of Mechanical Engineering and NETME Centre, regional research and development center established and founded from Operational Programme Research and Development for Innovation in a frame of project NETME Centre (New Technologies for Mechanical Engineering), Reg. no.: CZ.1.05/2.1.00/01.0002 and supported in a sustainability phase by project NETME CENTRE PLUS (LO1202) financed by The Ministry of Education, Youth and Sports in a frame of the "National sustainability program I".

\section{References}

1. F. A. Ribas, C. J. Deschamps, F. Fagotti, A. Morriesen and T. Dutra, International Compressor Engineering Conference, Paper 1907, (2008).

2. W. Lang, R. Almbauer, A. Burgstaller and D. Nagy, International Compressor Engineering Conference, Paper 1881, (2008).

3. R. Aigner and H. Steinrueck, 5th Conference of the EFRC, p.171-180, (2007). 
4. M. Costagliola, Journal of Applied Mechanics, vol. 17, p. 415-420, (1950).

5. Reinder André Habing, Flow and Plate Motion in Compressor Valves: Doctoral Thesis, (2005).

6. R. Ambauer, W. Lang, D. Nagy and E. Berger, International Compressor Egineering Conference, Paper 1968, (2010).

7. E. L. Pereira, C. J. Deschamps and F. A. Ribas, International Compressor Engineering Conference, Paper 1995, (2010).

8. F. P. Disconzi, C. J. Deschamps and E. L. Pereira,

International Compressor Engineering Conference, Paper 2103, (2012).

9. H. Steinrueck, R. Aigner and G. Machu, Acta Mechanica, vol. 201, p. 231-247, (2008). 